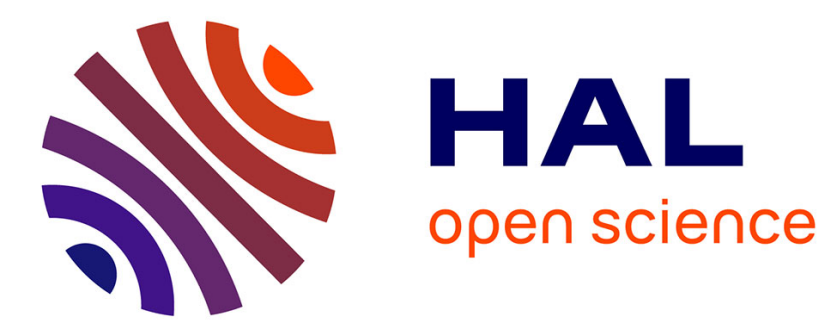

\title{
Dynamics of a chain of permanent magnets
}

\author{
Jean Boisson, Corinne Rouby, Joosung Lee, Olivier Doaré
}

\section{To cite this version:}

Jean Boisson, Corinne Rouby, Joosung Lee, Olivier Doaré. Dynamics of a chain of permanent magnets. EPL - Europhysics Letters, 2015, 109 (3), pp.34002. 10.1209/0295-5075/109/34002 . hal-01133414v2

\section{HAL Id: hal-01133414 \\ https://hal.science/hal-01133414v2}

Submitted on 26 Mar 2015

HAL is a multi-disciplinary open access archive for the deposit and dissemination of scientific research documents, whether they are published or not. The documents may come from teaching and research institutions in France or abroad, or from public or private research centers.
L'archive ouverte pluridisciplinaire HAL, est destinée au dépôt et à la diffusion de documents scientifiques de niveau recherche, publiés ou non, émanant des établissements d'enseignement et de recherche français ou étrangers, des laboratoires publics ou privés. 


\title{
Dynamics of a chain of permanent magnets
}

\author{
J. Boisson, C. Rouby, J. Lee, O. Doaré \\ Unité de Mécanique (UME), ENSTA Paristech, \\ 828 Boulevard des Maréchaux, 91762 Palaiseau cedex, France
}

PACS $45.50 .-\mathrm{j}$ - Dynamics and kinematics of a particle and a system of particles
PACS $45.20 . \mathrm{Jj}$ - Lagrangian and Hamiltonian mechanics
PACS $41.20 . \mathrm{Gz}$ - Magnetostatics; magnetic shielding, magnetic induction, boundary-value prob-
lems

\begin{abstract}
The dynamics of a straight chain of cylindrical neodymium magnets is considered. We showed this system exhibits a behavior which is similar to that of a beam where the elastic rigidity acts like a restoring force. By using a Lagrangian approach, a linear model, accounting for different sets of boundary conditions, is derived. Specifying to the clamped-free case eigenfrequencies and eigenmodes are determined. These theoretical results are compared to experiments. A good agreement is found, the discrepancies being attributed to the accumulation of defaults in the contact surface between the magnets. In the last part of the article, the equivalent beam behavior is sought for by defining an equivalent flexural rigidity induced by magnetic dipoles interactions. Published as: Jean Boisson, Corinne Rouby, Joosung Lee, Olivier Doaré. Dynamics of a chain of permanent magnets. EPL, Institute of Physics: EPL, 2015, 109 (3), pp.34002.
\end{abstract}

Introduction. - In the middle of the 1980's a new type of high energetic permanent magnet was developed $[1,2]$, based on neodymium alloys $\left(\mathrm{Nd}_{2} \mathrm{Fe}_{1} 4 \mathrm{~B}\right)$ designed by metallurgists [3]. Because of their strength and their small weight they enhanced the performances of many applications needing a magnetic field, like Magnetic Resonance Imagery [4], but also opened the possibility of a set of new applications in unexpected domain like medicine [5]. Because of their properties, the neodymium magnets can be used to build model experiments, where the magnets' collective behavior mimics those of microscopic systems (i.e. atoms interaction) at larger scale $[6,7]$.

The understanding of the collective behavior of magnetic particles has been the focus of a subsequent number of recent articles, with numerous applications such as colloidal particles chain behavior $[8,9]$, wave propagation in granular chains [10], magnetorheological fluid's behavior [11], propulsion of microorganisms [12], fluid transport and mixing [13], phase transition [14] or solitary wave propagation [15] in nonlinear lattices. The interactions of such particles embedded in a flexible material and their effect on the materials' behavior has also been the focus of researches [16].

Bonded magnets are manufactured from mixture of magnetic powders and binding resins [17]. These are structures with two distinct sources of stiffness: the resin elas- ticity and the magnetic interaction between magnetic particles.

In the literature one may distinguish between two kinds of dynamical models of magnetic dipole systems: a discrete description taking into account each dipole-dipole interaction $[6,7]$, or a continuous approach using a magnetization density [18]. In reference [19], the link between a continuous and a discrete description has been addressed. In these articles, the authors defined the equivalent bending rigidity of a chain of magnetic spheres by calculating the magnetic energy needed for bending a straight chain of magnet to form a ring. Such ring's dynamics was also studied in these papers. In the present article we focus on straight chains constituted by an assembly of cylindrical magnets. Although the statics of such systems was already studied $[11,18]$, to the authors knowledge, no results on the dynamics has been reported. Here, a horizontal chain of diametrically magnetized cylinders is considered. In its equilibrium state, the system forms a straight beam. The main objective of this paper is the study of eigenmodes and eigenfrequencies of this simple system.

The letter is organized as follows. First, the equations of motion of a chain of cylindrical dipoles are derived using Lagrange's formalism. Keeping only the first order terms, a set of linear dynamical equations are obtained. Next, a series of experiments is reported. Eigenfrequencies and 


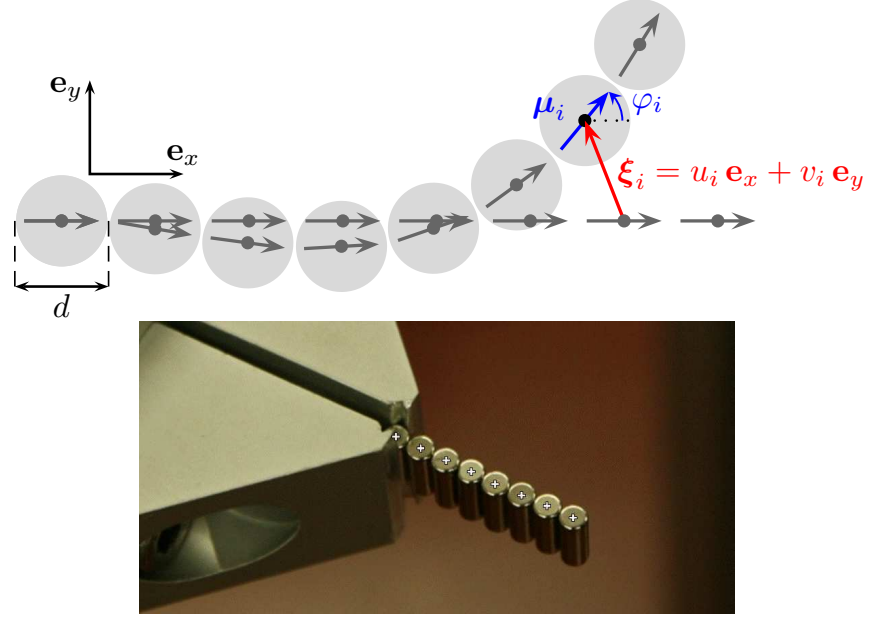

Fig. 1: Top: Sketch of a chain of magnetic dipoles at equilibrium and deformed configurations (here, $n=8$ ). Bottom: Photography of the experimental setup. The clamped is realized using aluminium triangles.

eigenmodes are obtained for various numbers of elements in the chain, of various sizes, mass and magnetic parameters. Experimental results are then compared with the model. Finally, this model is used to define an equivalent bending rigidity and the assumptions made in the model are discussed.

Equations of motion. - We consider the system sketched in Fig. 1, consisting of a chain of $n$ circular cylindrical magnets of diameter $d$ and mass $m$.

In the equilibrium configuration, the chain is straight and all dipoles are aligned along the $\mathbf{e}_{x}$ direction. In a deformed configuration, each dipole moves from its position in this reference configuration: the displacement of the center of the $i^{\text {th }}$ dipole is denoted by $\boldsymbol{\xi}_{i}$, and its moment, whose amplitude $\mu$ is unchanged but orientation may vary, by $\boldsymbol{\mu}_{i}$. The system is then described by $3 n$ generalized coordinates: the longitudinal and transverse displacements $u_{i}$ and $v_{i}$ and the orientation $\varphi_{i}$. These coordinates are not, however, independent variables. Indeed, each magnet is in contact with its neighbors and it is assumed that during the motion, the cylinders roll without slipping. This leads to the following holonomic constraints between the coordinates:

$$
\begin{aligned}
& g_{i}=u_{i+1}-u_{i}+d-d \cos \left(\frac{\varphi_{i}+\varphi_{i+1}}{2}\right)=0, \\
& h_{i}=v_{i+1}-v_{i}-d \sin \left(\frac{\varphi_{i}+\varphi_{i+1}}{2}\right)=0,
\end{aligned}
$$

for $i=1, \ldots, n-1$. This assumption will be justified in the discussion at the end of the letter by estimating the friction forces.

Finally, some displacements are imposed by the support conditions. Here, the clamped-free case is considered, thus the only boundary conditions are

$$
u_{1}=0 \quad, \quad v_{1}=0 \quad, \quad \varphi_{1}=0 .
$$

The parametrization of the system being performed, one shall write its Lagrangian $L$ as a function of both the generalized coordinates $u_{i}, v_{i}$ and $\varphi_{i}$ and their velocities $\dot{u}_{i}, \dot{v}_{i}$ and $\dot{\varphi}_{i}$. This function is defined as the difference between kinetic and magnetic energies of the system:

$$
L=\sum_{i=1}^{n} K_{i}-\frac{1}{2} \sum_{i=1}^{n} \sum_{\substack{j=1 \\ j \neq i}}^{n} U_{i j}
$$

In this expression, $K_{i}$ represents the kinetic energy of the $i^{\text {th }}$ dipole,

$$
K_{i}=\frac{1}{2} m\left(\dot{u}_{i}^{2}+\dot{v}_{i}^{2}\right)+\frac{1}{16} m d^{2} \dot{\varphi}_{i}^{2},
$$

and $U_{i j}$ the energy of magnetic interaction between the $i^{\text {th }}$ and $j^{\text {th }}$ dipoles [20],

$$
\begin{array}{r}
U_{i j}=-\frac{\mu_{0}}{4 \pi\left\|\mathbf{r}_{i j}\right\|^{3}}\left(\frac{3}{\left\|\mathbf{r}_{i j}\right\|^{2}}\left(\boldsymbol{\mu}_{i} \cdot \mathbf{r}_{i j}\right)\left(\boldsymbol{\mu}_{j} \cdot \mathbf{r}_{i j}\right)\right. \\
\left.-\boldsymbol{\mu}_{i} \cdot \boldsymbol{\mu}_{j}\right),
\end{array}
$$

where $\mu_{0}$ is the permeability of free space and $\mathbf{r}_{i j}$ is the vector joining the two dipoles, given by

$$
\mathbf{r}_{i j}=(j-i) d \mathbf{e}_{x}+\boldsymbol{\xi}_{j}-\boldsymbol{\xi}_{i} .
$$

Note that the cylindrical geometry of the magnets has been taken into account by writing the moment of inertia as $m d^{2} / 8$.

The Lagrange's equations of motion write [21]:

$$
\frac{\mathrm{d}}{\mathrm{d} t}\left(\frac{\partial L}{\partial \dot{q}}\right)-\frac{\partial L}{\partial q}=\sum_{i=1}^{n-1} \alpha_{i} \frac{\partial g_{i}}{\partial q}+\sum_{i=1}^{n-1} \beta_{i} \frac{\partial h_{i}}{\partial q},
$$

for $q=u_{k}, v_{k}, \varphi_{k}$ with $k=2, \ldots, n$. The evolution equations of the system are composed of the constraint equations (1) and the Lagrange's equations (7), with the boundary conditions (2). These represent a system of $5(n-1)$ differential equations, in which $3(n-1)$ generalized coordinates $u_{i}(t), v_{i}(t)$ and $\varphi_{i}(t)$, and $2(n-1)$ Lagrange's multipliers $\alpha_{i}(t)$ and $\beta_{i}(t)$, acting as reaction forces due to the constraint, are unknowns.

Nondimensional variables, denoted with overbars, are defined using the quantities $d, \sqrt{\pi m d^{5} / \mu_{0} \mu^{2}}$ and $\mu_{0} \mu^{2} / \pi d^{4}$ as characteristic length, time and force, respectively. Assuming small displacements and rotations, equations (1) and (7) are linearized. Within this approximation, longitudinal displacements $u_{i}$ are zero. The Lagrange's equations (7) corresponding to $q=u_{k}$ become:

$$
-\frac{3}{2} \sum_{\substack{i=1 \\ i \neq k}}^{n} \frac{i-k}{|i-k|^{5}}=\bar{\alpha}_{k-1}-\bar{\alpha}_{k}
$$

for $k=2, \ldots, n$. The multipliers $\bar{\alpha}_{i}$ are determined by inverting this linear system. 
Table 1: Experimental configurations and corresponding magnets characteristics. Magnetic poles are in gray and white.

\begin{tabular}{ccccc}
\hline Config. & Sketch & $d(\mathrm{~mm})$ & $m(\mathrm{~g})$ & $\mu\left(\mathrm{A} \mathrm{m}^{2}\right)$ \\
\hline a & $\rightarrow \rightarrow \rightarrow \rightarrow \rightarrow+$ & 4 & 0.930 & 0.068 \\
& & & & \\
$\mathrm{~b}$ & $\rightarrow \rightarrow \rightarrow \rightarrow \rightarrow \rightarrow+\rightarrow$ & 5 & 1.5 & 0.11 \\
\hline
\end{tabular}

After linearization, the constraint equations (1b) become

$$
\bar{v}_{k+1}-\bar{v}_{k}=\frac{1}{2}\left(\varphi_{k}+\varphi_{k+1}\right)
$$

for $k=1, \ldots, n-1$. From these equations and the boundary conditions $(2)$, the $2 n$ generalized coordinates $\bar{v}_{i}$ and $\varphi_{i}$ are reduced to $n-1$ independent variables $\eta_{i}$. The general coordinates are then functions of these independent variables: $\bar{v}_{i}(\boldsymbol{\eta})$ and $\varphi_{i}(\boldsymbol{\eta})$ where $\boldsymbol{\eta}$ is the vector of size $n-1$ gathering the values of $\eta_{i}$. In the clampedfree case exhibited here, these variables can be defined as $\eta_{i}=\bar{v}_{i+1}$. This definition must be adapted when other boundary conditions are considered. The number of independent variables, corresponding to the system's degrees of freedom, may also be modified.

Lagrange's equations (7) corresponding to $q=v_{k}$ and $q=\varphi_{k}$ lead to a linear system of $2(n-1)$ equations in $2(n-1)$ unknowns $\eta_{i}$ and $\bar{\beta}_{i}$, which reads:

$$
\text { see eq. (10) }
$$

for $k=2, \ldots, n$. Performing linear combinations of these equations in order to eliminate $\bar{\beta}_{i}$ leads to a linear system of the form

$$
\mathbf{M} \ddot{\boldsymbol{\eta}}+\mathbf{K} \boldsymbol{\eta}=0
$$

Eigenmodes and eigenfrequencies of the system are then obtained by diagonalization. It must be noted that in its dimensionless form, these equations of motion depend only on one parameter: the number of dipoles $n$.

Experiments. - Experiments are performed with two configurations of magnet chain. In configuration (a), each cylinder of the chain is composed by a single magnet, while in configuration (b), a pair of magnets arranged along the cylinder axis and with inverted magnetic poles constitutes the basic element of the chain, as sketched in Tab. 1. A magnet consists of neodymium, iron and boron alloy and are plated by nickel-copper. $t$ is known that cylinders, contrary to spheres, do not have a perfect dipolar behavior [22]. This has been confirmed by our measurements of the attractive force between two magnets at different distances and orientations. However, we observed that the short scale interactions can be very well approximated by a dipolar approximation of the magnets, if we tolerate an small error in the far field. Since the force is rapidly decaying as $1 / r^{4}$, the error is negligible. Hence, the
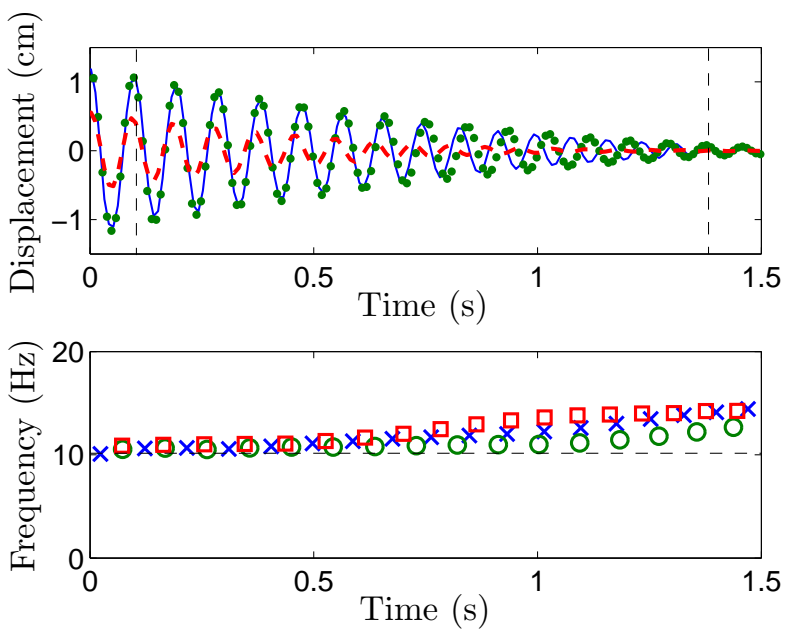

Fig. 2: Top: Displacement of the fourth cylinder within a $n=6$ chain in configuration (a) of Tab. 1. The three different curves correspond to three typical experiments. Bottom: Oscillation frequency of each cycle obtained by tracking zero-crossings of experimental data reported above, where $(\times, \square, \circ)$ correspond respectively to $(-,--,, \cdots)$ of the top figure. Horizontal dashed line: frequency predicted by the linear model. Left and right vertical dashed lines correspond to the left and right plots of Fig. 3 respectively.

short range interactions forces measured experimentally are used to model the magnets as dipoles with magnetic moment $\mu=0.068 \pm 0.003 \mathrm{~A} \mathrm{~m}^{2}$ in the configuration (a) and $\mu=0.12 \pm 0.01 \mathrm{~A} \mathrm{~m}^{2}$ in the configuration (b).

Dynamics of structures from 2 to 21 cylinders is considered, the first cylinder being clamped, as modeled in the previous section. Experimentally, the clamping is performed using two triangles holding the first cylinder along its axis. These configurations restrict the motion in the horizontal plane to avoid the influence of gravity on the dynamics (see Fig. 1).

Free oscillations of the chain is now considered. The system is excited by moving the $n^{\text {th }}$ cylinder away from its equilibrium position and releasing it with no initial velocity. With such type of initial condition, we expect the oscillation to be dominated by the first linear mode. Moreover, at moderate amplitudes, it is expected that nonlinearities do not affect the dynamics. We recorded the free oscillations decays for chain lengths from 2 to 21 elements using a high speed camera. The chosen sampling rate was of at least 20 points per period. Using an image processing involving opening and closing filters, positions of the centers of the cylinders top surfaces were tracked, allowing us to reconstruct the trajectory of each cylinder during a decay. The oscillating frequency is then obtained by tracking zero-crossings in the time series.

Fig. 2 displays the results of three typical experimental runs for a chain of configuration (a) with 6 elements. For two of these runs, the initial conditions are the same, while the initial displacement is divided by two in the third run. 


$$
\begin{aligned}
& \ddot{\bar{v}}_{k}-3 \sum_{\substack{i=1 \\
i \neq k}}^{n} \frac{1}{|i-k|^{5}}\left(\bar{v}_{i}-\bar{v}_{k}\right)+\frac{3}{4} \sum_{\substack{i=1 \\
i \neq k}}^{n} \frac{i-k}{|i-k|^{5}}\left(\varphi_{i}+\varphi_{k}\right)=\bar{\beta}_{k-1}-\bar{\beta}_{k} \\
& \ddot{\varphi}_{k}-6 \sum_{\substack{i=1 \\
i \neq k}}^{n} \frac{i-k}{|i-k|^{5}}\left(\bar{v}_{i}-\bar{v}_{k}\right)+2 \sum_{\substack{i=1 \\
i \neq k}}^{n} \frac{1}{|i-k|^{3}}\left(\varphi_{i}+2 \varphi_{k}\right)=2 \bar{\alpha}_{k-1}\left(\varphi_{k-1}+\varphi_{k}\right)+2 \bar{\alpha}_{k}\left(\varphi_{k}+\varphi_{k+1}\right)-4 \bar{\beta}_{k-1}-4 \bar{\beta}_{k},
\end{aligned}
$$

In all reported measurements, oscillation frequencies for $t<0.75 \mathrm{~s}$ are around $10 \mathrm{~Hz}$, which is the value predicted by the model. However, at longer times, all measurements depart from each other and a gradual increase of the oscillation frequencies is observed. This behavior, exhibited here for the particular case of 6 cylinders, is recurrent for all values of $n$.

At least two nonlinear phenomena may explain this frequency increase. A first assumption would be to consider geometrical nonlinearities. However this shall be discarded because the evolution of the frequency with the amplitude is not reproducible, like that observed in Fig. 2.

Another nonlinear phenomenon may then be responsible of the discrepancy with the linear model: the accumulation of impurities on the cylinders and surface defects on the contact line. The size of these contact defaults are of the order of $0.1 \mathrm{~mm}$. Thus they should influence the motion when the amplitude of the contact line displacement is of the same order, i.e. for the small amplitudes. This is confirmed by the smaller increase of the frequency when brand new sets of cylinders are used (symbols $\circ$ of Fig. 2). As an illustration, the position of the chain is represented in Fig. 3 for the three runs at two different instants. These are marked by vertical dashed line in Fig. 2: at $t \sim 0.1 \mathrm{~s}$ and at $t \sim 1.4 \mathrm{~s}$. The displacements of the dipoles are compared to the first linear eigenmode predicted by the model. A good agreement exists for the three runs at $t \sim 0.1 \mathrm{~s}$. Conversely, at $t \sim 1.4 \mathrm{~s}$, the experimental mode departs from the theoretical one. We assume that impurities seize up the junction between cylinders, and induce an additional stiffness which is responsible of this discrepancy. Due to this frequency shift behavior, and the discrepancy of eigenmodes, we estimate frequencies measured on the first oscillations. Full comparison with the linear model is done at the end of this section.

The configuration (a) of Tab. 1 is now clamped on a rigid aluminium rod of $30 \mathrm{~cm}$ length. The rod is fixed on a electrodynamic shaker, so that the first dipole of the chain has displacement of the form $v_{1}(t)=A \sin \left(\omega_{f} t\right)$, where $\omega_{f}$ is the prescribed oscillations frequency. The presence of the aluminium bar prevents electromagnetic interactions between the magnets and the shaker. As for free vibrations experiments, the chain oscillations plane is horizontal to avoid influence of gravity on the dynamics.

The chain of cylindrical magnets is forced using a linear up and down frequency sweep in the range $[1-40] \mathrm{Hz}$. Simultaneously, the displacement of the chain at $3 / 4$ of its

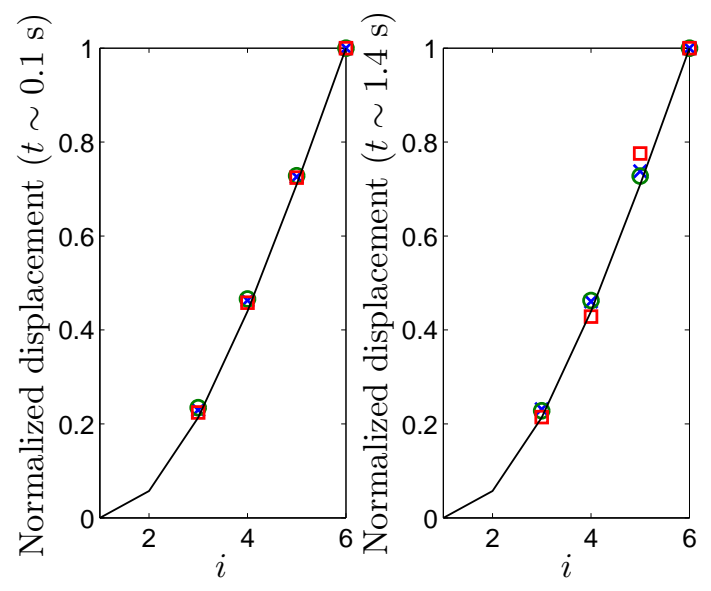

Fig. 3: (-), first theoretical eigenmode for $n=6$, normalized by the displacement of the $n^{t h}$ dipole; $(\times, \square, \circ)$, displacement of each individual cylinder measured when the chain reaches a maximum of displacement. Left: $t \sim 0.1$ s, i.e. second maximum of Fig. 2(top). Right: maximum of displacement around $t \sim 1.4 \mathrm{~s}$, after 17 oscillations. Both times are marked by vertical lines in the top of Fig. 2 .

length is tracked with a displacement sensor. Fig. 4 displays transfer functions between displacement of the first dipole and the one measured by the sensor for $n=6$ and $n=10$. On the same figure, eigenfrequencies predicted by the model are represented. An overall agreement is found between peaks of the transfer functions and theoretical eigenfrequencies with a relative error in the range $[4-15] \%$. For the data plotted on Fig. 4, the amplitude of the response is of the order of $1 \mathrm{~cm}$, which corresponds to the first oscillations of Fig. 3. Smaller excitation amplitudes have also been tested and similarly to the case of free oscillations, an increase of the frequencies is observed. Again, we attribute this effect to contact defaults, that we have not modeled. Moreover we observe almost no jump phenomena or hysteresis between the up and down sweep direction.

The first two experimental eigenfrequencies are compared to the model on Fig. 5. Results for the two different cylinders arrangements and two different measurement methods are exhibited. One can observe a good agreement between experiments and theory. On this figure, the asymptotic behavior for large $n$ is also represented: the eigenfrequencies vary as $n^{-2}$. The length used to obtain the dimensionless equation is the diameter $d$, hence the 

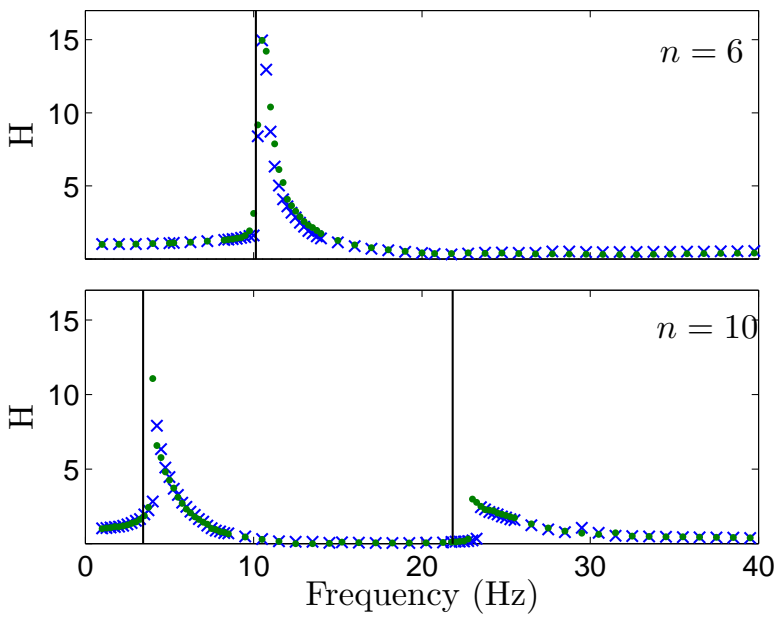

Fig. 4: Modulus of the transfer function between displacement of the clamped cylinder and the cylinder at $3 / 4$ of the length of the chain. Each mark corresponds to an average over 20 seconds. The $(\times, \cdot)$ symbols represent the up and down sweep direction respectively. The vertical lines are the linear eigenfrequencies predicted by the model.

eigenfrequencies vary as the inverse of the total length squared. The implications of this observation will be discussed in the next section.

Discussion. - In Fig. 5, both numerical and experimental data allow us to conclude that when the number of dipoles $n$ is large, eigenfrequencies are proportional to the inverse of the chain length square. This is a typical behavior for beams. Elasticity of beams is characterized by the flexural rigidity $K$ (in beams, the flexural rigidity is classically denoted $E I$, where $E$ is the Young's modulus and $I$ the moment of inertia of the cross sections). Due to long distance interactions, magnetic forces between each dipole induce restoring forces which are more complex than a purely elastic bending restoring force. However, we seek here to approach the macroscopic chain behavior through a unique flexural rigidity coefficient $K_{\text {eq }}$. The latter is estimated calculating the flexural rigidity which gives the same eigenfrequency for a given mode. The $i^{\text {th }}$ circular eigenfrequency of a clamped-free beam reads [23]

$$
\omega_{i}^{2}=\frac{\lambda_{i}^{4}}{l^{4}} \frac{K}{\rho},
$$

where $\rho$ is the mass per unit length of the beam, $l$ its length and $\lambda_{i}$ is the $i^{\text {th }}$ solution of the characteristic equation $\cos \lambda \cosh \lambda+1=0$. Using parameters of the present problem, the equivalent flexural rigidity can be written in the following form:

$$
\bar{K}_{\mathrm{eq}}=K_{\mathrm{eq}} \frac{\pi d^{2}}{\mu_{0} \mu^{2}}=\left(\frac{n-1}{\lambda_{i}}\right)^{4} \bar{\omega}_{i}^{2} .
$$

The quantity $\bar{K}_{\mathrm{eq}}$ is plotted in the inset of Fig. 6 as function of $n-1$ for the three first modes. Although slight

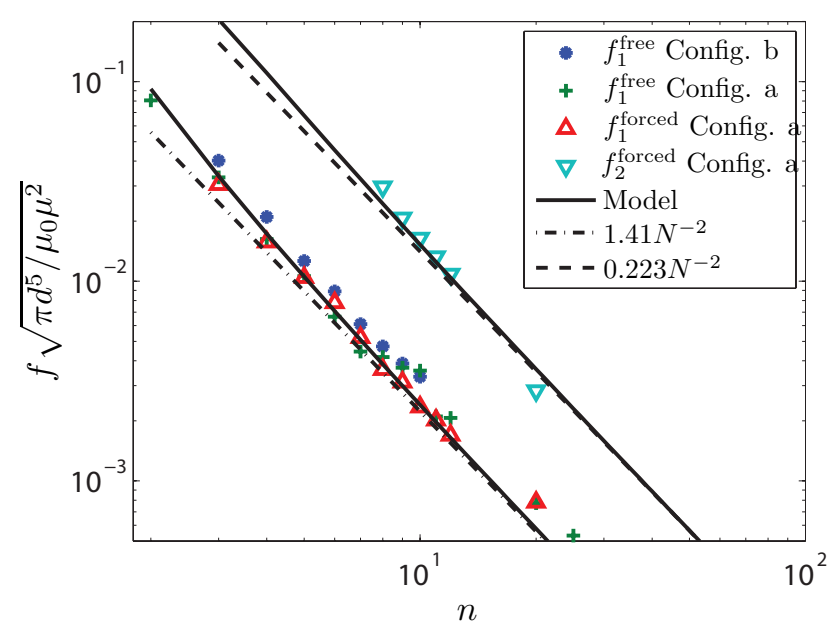

Fig. 5: Comparison of experimental and theoretical eigenfrequencies. Symbols represent experimental results for different cases, plain lines show the two first theoretical eigenfrequencies, dashed and dash-dotted lines are the asymptotic behavior of theoretical eigenfrequencies for large $n$.

differences exist, the same trend can be observed for the three curves: a monotonic increase of $\bar{K}_{\text {eq }}$ and a convergence to a limit value around 0.16 . Let us define the limit value $\bar{K}_{\infty}=\lim _{n \rightarrow \infty} \bar{K}_{\text {eq }}$. This quantity is then plotted as function of the mode number on Fig. 6. For completeness, the same quantity is plotted in the pinned-pinned and clamped-clamped cases.

For large values of the mode number, $\bar{K}_{\infty}$ converges to $\sim 0.171$. In reference [6], the energy of a circular ring of magnetic spheres was calculated and its asymptotic value when the number of spheres reaches infinity was utilized to calculate an equivalent bending rigidity as $K_{\text {eq }} \sim 0.239 B^{2} d^{4} / 16 \mu_{0}$, where $B$ is the magnetic strength of the magnet. Expressed in the parameters' set of the present article, this gives $K_{\text {eq }} \sim 0.171 \mu_{0} \mu / \pi d^{2}$ and is represented as a dashed line in Fig. 6. It can then be observed that the same value is obtained here, but only for higher modes. The fact that a smaller equivalent bending rigidity is found for lower modes can be explained by side effects. Indeed, as the mode number increases all modes approach a sinusoidal shape except at boundaries. Hence the number of sinusoidal oscillations contained in the mode increases and boundary effects see their influence decreasing. Note that an equivalent bending rigidity has also been found in the case of a continuous filament in an applied magnetic field [24].

Lagrange's multipliers, which can be calculated for a given system evolution, represent reaction forces due to the constraints. Thus, the contact force exerted by the $i^{\text {th }}$ dipole on the $(i+1)^{\text {th }}$ dipole is $\alpha_{i} \mathbf{e}_{x}+\beta_{i} \mathbf{e}_{y}[21]$. At each contact point, the normal force $N_{i}$ and the friction force $T_{i}$ are the components of this contact force which are parallel and perpendicular to the line joining the two dipoles. According to Coulomb's law, there is no slipping 


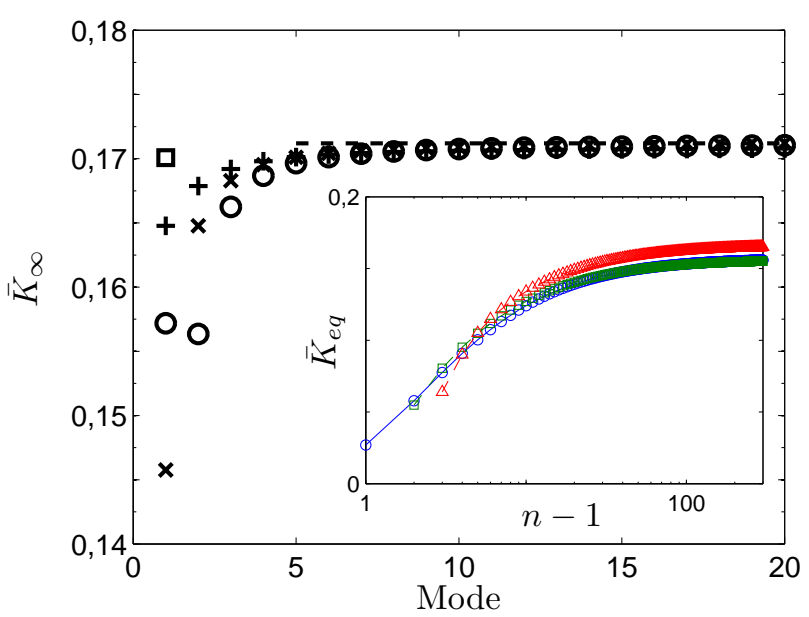

Fig. 6: Normalized equivalent bending rigidity for $n \rightarrow \infty$ as function of the mode number; (o), clamped-free boundary conditions; $(\times)$, pinned-pinned boundary conditions; $(+)$, clamped-clamped boundary conditions; $(---)$, value predicted in reference [6] for a ring. Inset : Equivalent bending rigidity normalized by $\mu_{0} m^{2} / \pi D^{2}$ as function of $n-1$; (o), mode $1 ;(\square)$, mode $2 ;(\Delta)$; mode 3 .

between the two dipoles while the ratio

$$
\frac{T_{i}}{N_{i}} \simeq \frac{\beta_{i}}{\alpha_{i}}-\left(1+\left(\frac{\beta_{i}}{\alpha_{i}}\right)^{2}\right)\left(\bar{v}_{i+1}-\bar{v}_{i}\right)
$$

is lower than the coefficient of friction. For nickel-nickel contacts, this coefficient is $\sim 0.3$.

The ratio in equation (14) depends only on non dimensional parameters of the problem. Let us consider the displacement of the chain along a single eigenmode at a maximum amplitude of displacement of half the length. Under these circumstances, for modes 1 and 2 and for any number of dipoles in the chain, it has been verified with the model that the ratio $T_{i} / N_{i}$ never reaches the friction coefficient, thus validating the rolling assumption.

Conclusion. - In this article, the linear dynamics of a chain of magnetic dipoles has been characterized theoretically and experimentally. First, the linear dynamics has been described in the form of an eigenvalue problem. Next, experiments in the clamped-free setup have been performed and experimental eigenfrequencies have been successfully compared to the theoretical ones. Finally, the chain has been described in terms of an equivalent beam. An equivalent bending rigidity has been computed for each mode and it is found that when the number of dipoles becomes large, it scales as $\bar{K} \mu_{0} \mu^{2} / \pi d^{2}$, where $\bar{K}$ is comprised between 0.145 and 0.171. For higher order eigenmodes, whatever the boundary conditions we applied, $\bar{K}$ tends to the value found in reference [6] where the energy of a circular ring was calculated. The difficulty introduced by using magnets has been demonstrated. Indeed, impurities that accumulate on the contact lines between two consec- utive cylinders introduced discrepancies between theory and experiments that are difficult to get rid of. Once this experimental difficulties have been treated, future work on such systems may include non linear effects, other configurations, or other boundary conditions pointing to mechanical behavior which cannot be described by classic elastic theory. The study of similarities between this discrete system and continuous structures permanently magnetized [18] is also of great interest.

This work is supported by the Fédération Francilienne de Mécanique (F2M-msp) and by a public grant overseen by the French National research Agency (ANR) as part of the "Investissement dAvenir" program, through the "IDI 2013" project funded by the IDEX Paris-Saclay, ANR-11IDEX-0003-02

\section{REFERENCES}

[1] Croat J. J., Herbst J. F., Lee R. W. and Pinkerton F. E., Appl. Phys. Lett., 44148 (1984)

[2] Sagawa M., Fujimura S., Togawa N., Yamamoto H. and Matsuura Y., J. Appl. Phys., 552083 (1984)

[3] Chaban N. F., Kuz'ma Y. B., Bilonizhko N. S., KaChmar O. O. and Petriv N. V., Dopovidi Akad. Nauk Ukr. RSR, Ser. A, 10873 (1979)

[4] Livingston. J. D., MRS Bull., 2155 (1996)

[5] Noar J. H. and Evans R. D., J. Orthodontics, 2629 (1999)

[6] Vella D., du Pontavice E., Hall C. L. and Goriely A., Proc. Roy. Soc. A, 47020130609 (2014)

[7] Vanderwalle N. and Dorbolo S., New J. Phys., 16 013050 (2014)

[8] Coq N., Ngo S., du Roure O., Fermigier M. and BarTOlO D., Phys. Rev. E, 82041503 (2010)

[9] Cerdà J. J., Sánchez P. A., Holm C. and Sintes T., Soft Matter, 97185 (2013)

[10] Cabaret J., Tournat V. and Béquin P., Phys. Rev. E, 86041305 (2012)

[11] Biswal S. L. and Gast A. P., Phys. Rev. E, 69041406 (2004)

[12] Shcherbakov V. P., Winklhofer M., Hanzlik M. and Petersen N., Eur. Biophys. J., 26319 (1997)

[13] Gauger E. M., Downton M. T. and Stark H., Eur. Phys. J. E, 28231 (2009)

[14] Shutyi A. M., Tech. Phys+, 59325 (2014)

[15] Molerón M., Leonard A. and Daraio C., J. Appl. Phys., 115184901 (2014)

[16] Danas K., Kankanala S. V. and Triantafyllidis N., J. Mech. Phys. Solids, 60120 (2012)

[17] Ormerod J. and Constantinides S., J. Appl. Phys., 81 4816 (1997)

[18] Cḗbers A. and Cīrulis T., Phys. Rev. E, 76031504 (2007)

[19] Hall C. L., Vella D. and Goriely A., SiAM J. Appl. Math., 732029 (2013)

[20] JaCKson J. D., Classical Electrodynamics (Wiley)1998 
[21] Landau L. D. and Lifshitz E. M., Course of Theoretical Physics, Vol. 1 (Pergamon Press) 1969

[22] Petruska A. J. and Аввотt J. J., Ieee T. Magn., 49 811-819 (2013)

[23] Blevins R. D., Formulas for natural frequency and mode shape (Van Nostrand Reinhold Co., New York)1979

[24] CĒBers A., Curr. Opin. Colloid In., 10 167-175 (2005) 